\title{
SOLUTIONS OF THE NONLINEAR DIFFERENTIAL EQUATIONS BY USE OF SYMMETRIC FIBONACCI FUNCTIONS
}

\author{
Yusuf Pandir $^{1}$, Yusuf Ali Tandogan ${ }^{2}$ \\ Department of Mathematics \\ Faculty of Arts and Science \\ Bozok University \\ Cemil Cicek Avenue 7th miles Erdogan Akdag Campus \\ Yozgat, 66100, TURKEY
}

\begin{abstract}
Based on Kudryashov's method and the Fibonacci or Lucas Riccati equation method, some new solutions of a non-integrable nonlinear partial differential equation are found. Also, some basic properties of symmetric Fibonacci and Lucas functions are given in this research. For more details, we refer the reader to [1]-[5].
\end{abstract}

AMS Subject Classification: 35C08, 35Q51, 35Q53

Key Words: Kudryashov's method, variable coefficient of Korteweg-de Vries $(\mathrm{KdV})$ equation, symmetric Fibonacci functions, exact solutions

\section{Introduction}

In the past few decades there has been ongoing research in various kinds of nonlinear evolution equations. Several various methods [6]-[13] have been used to handle nonlinear equations with constant coefficients and with time dependent coefficients. We consider the variable coefficient of Korteweg-de Vries (KdV) equation with higher-order nonlinearity

Received: June 19, 2013

(C) 2013 Academic Publications

${ }^{\S}$ Correspondence author 


$$
u_{t}(x, t)+\sigma(t) u^{p}(x, t) u_{x}(x, t)+\delta(t) u_{x x x}(x, t)=0,
$$

where $\sigma(t), \delta(t)$ are functions depending of the time $t$ and $p$ is a positive number. Many authors have studied some types of solutions of this equation. There are many methods that are used to obtain the integration of nonlinear partial differential equations. Some of them are the exp-function method [7][10], the trial equation method [11], the $\left(G^{\prime} / G\right)$-expansion method, the Hirota's method [14], the auxiliary equation method [15], and many more. Our aim is deriving a new solutions to Eq. (1) using the improved Kudryashov method [2]. In this research, we modify Kudryashov's method (Kudryashov, 2012) to raise the effectiveness of this method. Our key idea is that traditional base $e$ of the exponential function is replaced by an arbitrary base $a \neq 1$. So, new exact solutions of nonlinear evolution equations may be obtained by this simple modification.

\section{The Modified Kudryashov Method}

We consider the following nonlinear partial differential equation for a function $q$ of two real variables, space $x$ and time $t$ :

$$
P\left(t, x, q, q_{t}, q_{x}, q_{t t}, q_{t x}, q_{x x}, \ldots\right)=0 \text {. }
$$

It is useful to summarize the main steps of modified Kudryashov method as follows:

Step 1. We seek the travelling wave solution of Eq. (2) of the form

$$
q(x, t)=u(\xi), \quad \xi=k x+\int^{t} w\left(t^{\prime}\right) d t^{\prime},
$$

where $k$ is a free constant. We reduce Eq. (2) to a nonlinear ordinary differential equation of the form:

$$
N\left(t, x, u, u^{\prime}, u^{\prime \prime}, \ldots\right)=0,
$$

where the prime denotes differentiation with respect to $\xi$. Suppose that the highest order nonlinear terms in Eq. (4) are $u^{l}(\xi) u^{(s)}(\xi)$ and $\left(u^{(p)}\right)^{k}$.

Step 2. We suppose that the exact solutions of Eq. (4) can be obtained in the following form:

$$
u(\xi)=y(\xi)=\sum_{j=0}^{N} a_{j}(t) Q^{j}
$$


where $Q=\frac{1}{1 \pm a^{\xi}}$. We note that the function $Q$ is solution of equation

$$
Q_{\xi}=\ln a\left(Q^{2}-Q\right)
$$

Step 3. According to the proposed method, we assume that the solution of Eq. (4) can be expressed in the form

$$
u(\xi)=a_{N} Q^{N}+\ldots
$$

Calculation of value $N$ in formula (7) that is the pole order for the general solution of Eq. (4). To determine the value of $N$ we proceed analogously as in the classical Kudryashov method on balancing the highest order nonlinear terms in Eq. (4). More precisely, by straightforward calculations we have

$$
\begin{gathered}
u^{\prime}(\xi)=a_{N} N Q^{N+1}+\ldots, \\
u^{\prime \prime}(\xi)=a_{N} N(N+1) Q^{N+2}+\ldots, \\
u^{(s)}(\xi)=a_{N} N(N+1) \ldots(N+s-1) Q^{N+s}+\ldots, \\
u^{l} u^{(s)}(\xi)=\overline{a_{N}} N(N+1) \ldots(N+s-1) Q^{(l+1) N+s}+\ldots, \\
\left(u^{(p)}\right)^{k}(\xi)=\left(a_{N} N(N+1) \ldots(N+s-1)\right)^{k} Q^{k(N+p)}+\ldots,
\end{gathered}
$$

where $a_{N}$ and $\overline{a_{N}}$ are constant coefficients. Balancing the highest order nonlinear terms of Eq. (11) and Eq. (12), we have

$$
(l+1) N+s=k(N+p),
$$

so

$$
N=\frac{s-k p}{k-l-1}
$$

Step 4. Substituting Eq. (5) into Eq. (4) yields a polynomial $R(Q)$ of $Q$. Setting the coefficients of $R(Q)$ to zero, we get a system of algebraic equations. Solving this system, we shall determine $w(t)$ and the variable coefficients of $a_{0}(t), a_{1}(t), \ldots, a_{N}(t)$. Thus, we obtain the exact solutions to Eq. (2). 


\section{Application of the Method to Variable Coefficient of KdV Equation}

We first assume that Eq. (1) has solutions of the form

$$
u(x, t)=v(\xi), \quad \xi=k x+\int^{t} q\left(t^{\prime}\right) d t^{\prime}
$$

and substituting it into Eq. (1), we can reduce to the ordinary differential equation which can be written as

$$
q(t) v^{\prime}(\xi)+k \sigma(t)(v(\xi))^{p} v^{\prime}(\xi)+k^{3} \delta(t) v^{\prime \prime \prime}(\xi)=0 .
$$

Upon integration, Eq. (3) is converted to

$$
(p+1) k^{3} \delta(t) v^{\prime \prime}(\xi)+(p+1) q(t) v(\xi)+k \sigma(t) v^{p+1}(\xi)=C,
$$

where $C$ is the integration constant. For simplicity we take $C=0$ and use the transformation

$$
v(\xi)=V^{\frac{1}{p}}(\xi)
$$

which will convert to Eq. (4) into

$$
\begin{aligned}
k^{3} p\left(p^{2}+1\right) \delta(t) V(\xi) V^{\prime \prime}(\xi)+k^{3}(1- & \left.p^{2}\right) \delta(t)\left(V^{\prime}(\xi)\right)^{2} \\
& +\left(p^{3}+p^{2}\right) q(t) V^{2}+k p^{2} \sigma(t) V^{3}=0 .
\end{aligned}
$$

We take

$$
V(\xi)=y(\xi)=\sum_{n=0}^{N} a_{n} Q^{N}
$$

where $Q=\frac{1}{1 \pm a^{\xi}}$. We note that the function $Q$ is solution of equation

$$
Q_{\xi}=\ln a\left(Q^{2}-Q\right)
$$

Considering the homogeneous balance with $V V^{\prime \prime}$ and $V^{3}$ in Eq. (6) gives

$$
\begin{gathered}
2 N+2=3 N, \\
N=2 .
\end{gathered}
$$

Therefore we have

$$
V(\xi)=y(\xi)=\sum_{N=0}^{2} a_{N} Q^{N}=a_{0}+a_{1} Q+a_{2} Q^{2},
$$


and we substitute derivatives of the function $y(\xi)$ with respect to $\xi$. The required derivatives in Eq. (6) are obtained

$$
\begin{gathered}
y_{\xi}=\ln a\left(-a_{1} Q+a_{1} Q^{2}-2 a_{2} Q^{2}+2 a_{2} Q^{3}\right), \\
y_{\xi \xi}=\ln ^{2} a\left(a_{1} Q-3 a_{1} Q^{2}+2 a_{1} Q^{3}+4 a_{2} Q^{2}-10 a_{2} Q^{3}+6 a_{2} Q^{4}\right) .
\end{gathered}
$$

As a result of this we have the system of algebraic equations can be solved with Mathematica. Solving the systems we obtain coefficients $a_{0}(t), a_{1}(t)$ and $a_{2}(t)$ as follows:

$$
\begin{gathered}
a_{0}(t)=0, \quad a_{1}(t)=\frac{2 k^{2} \ln a^{2}\left(2+3 p+p^{2}\right) \delta(t)}{p^{2} \sigma(t)} \\
a_{2}(t)=-\frac{2 k^{2} \ln a^{2}\left(2+3 p+p^{2}\right) \delta(t)}{p^{2} \sigma(t)}, \quad q(t)=-\frac{k^{3} \ln a^{2} \delta(t)}{p^{2}},
\end{gathered}
$$

where $\delta(t)$ and $\sigma(t)$ are arbitrary functions. Substituting Eq. (14) into (11) and we have

$$
\begin{aligned}
V(\xi)=\frac{2 k^{2} \ln a^{2}\left(2+3 p+p^{2}\right) \delta(t)}{p^{2} \sigma(t)} & \frac{1}{1 \pm a^{\xi}} \\
- & \frac{2 k^{2} \ln a^{2}\left(2+3 p+p^{2}\right) \delta(t)}{p^{2} \sigma(t)} \frac{1}{\left(1 \pm a^{\xi}\right)^{2}},
\end{aligned}
$$

where $\xi=k\left(x-\int^{t} \frac{k^{2} \ln a^{2} \delta\left(t^{\prime}\right)}{p^{2}} d t^{\prime}\right)$. Therefore, we obtain the following solutions of $\mathrm{Eq}(1)$

$$
\begin{aligned}
& u_{1}(x, t)=\frac{A_{1}(t)}{\operatorname{cFs}^{\frac{2}{p}}[B(x-v(t))]}, \\
& u_{2}(x, t)=\frac{A_{2}(t)}{\operatorname{sFs}^{\frac{2}{p}}[B(x-v(t))]},
\end{aligned}
$$

where $A_{\ni}(t)=\left(\frac{2(-1)^{\ni} k^{2} \ln a^{2}\left(2+3 p+p^{2}\right) \delta(t)}{5 p^{2} \sigma(t)}\right)^{\frac{1}{p}},(\ni=1,2),. \quad B=\frac{k}{2}$ and $v(t)=$ $\int \frac{k^{2} \ln a^{2} \delta\left(t^{\prime}\right)}{p^{2}} d t^{\prime}$. Here, $A_{1}(t), A_{2}(t)$ represent the amplitude of the solitons, while $B$ is the inverse width of the solitons and $v=v(t)$ represent the velocity of the solitons. Figures 1-3 show the solutions $u_{1}(x, t), u_{2}(x, t)$ for the values $k=p=1$, the functions $\delta(t), \sigma(t)$ and $a$ takes respectively, Golden Mean, $e$ and 10 . 

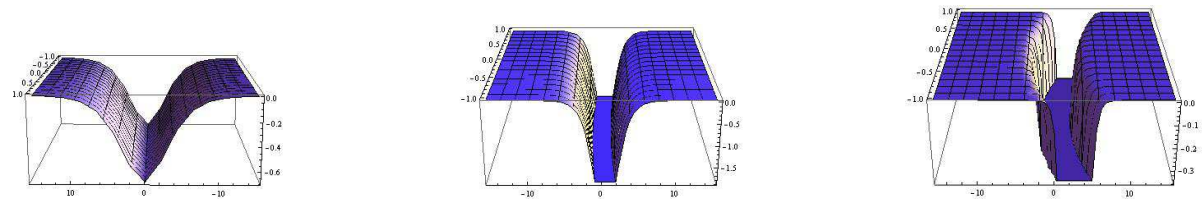

Figure 1: Solution of $u_{1}(x, t)$ is shown at $k=p=1, \delta(t)=\sigma(t)=t$.
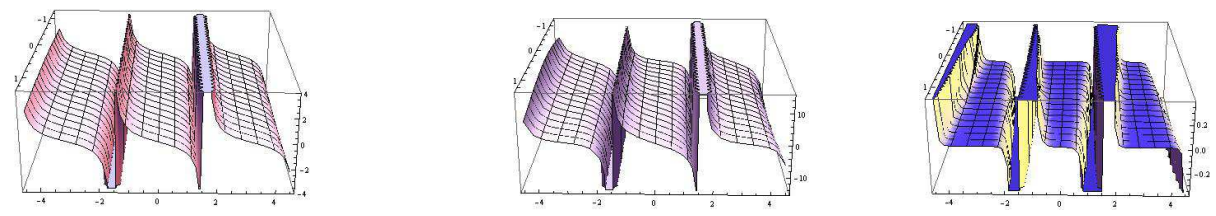

Figure 2: Solution of $u_{1}(x, t)$ is shown at $k=p=1, \delta(t)=$ $\sin (t), \sigma(t)=\cos (t)$.
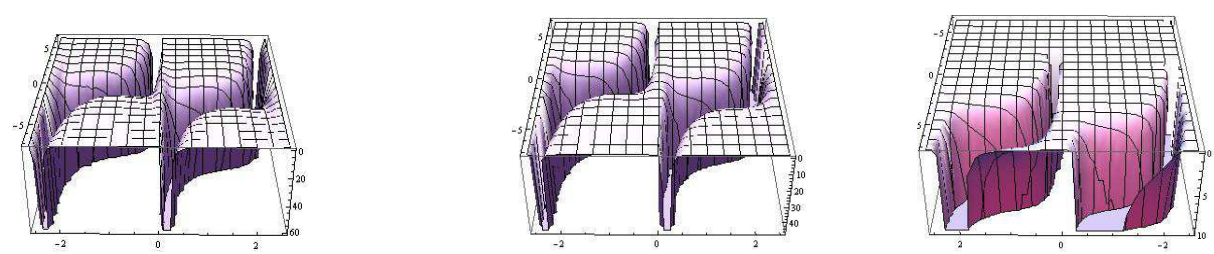

Figure 3: Solution of $u_{2}(x, t)$ is shown at $k=1, p=1$, $\delta(t)=$ Weierstrass elliptic function $[\mathrm{t}, 1 / 3], \quad \sigma(t)=1$. 


\section{Remarks and Conclusion}

Our aim in this section is to show that general $E x p_{a}$-function with Kudryashov's method could be used to one solutions in the form of symmetrical hyperbolic Fibonacci and Lucas functions. We highlight briefly the definitions of symmetrical hyperbolic Fibonacci and Lucas functions. Also Stakhov and Rozin [4] defined all details of symmetrical hyperbolic Fibonacci and Lucas functions. We only give formulas here. Symmetrical Fibonacci sine, cosine and tangent are respectively defined as

$$
s F s(x)=\frac{a^{x}-a^{-x}}{\sqrt{5}}, \quad c F s(x)=\frac{a^{x}+a^{-x}}{\sqrt{5}}, \quad t F s(x)=\frac{a^{x}-a^{-x}}{a^{x}+a^{-x}} .
$$

Analogously, symmetrical Lucas sine and cosine are respectively defined as

$$
s L s(x)=a^{x}-a^{-x}, \quad c L s(x)=a^{x}+a^{-x},
$$

where $a=\frac{1+\sqrt{5}}{2}$, which is known in the literature as Golden Mean [1]. So we can find more general (or more larger classes of) solutions in applying the general Exp-function method with symmetrical Fibonacci functions. If we take $a=e$ then we can find other solutions also.

\section{References}

[1] T.A. Ahmad and R.H. Ezzat, General Exp ${ }_{a}$-function method for nonlinear evolution equations, Appl. Math. Comput., 217 (2010), 451-459.

[2] N.A. Kudryashov, One method for finding exact solutions of nonlinear differential equations, Commun. Nonl. Sci. Numer. Simulat., 17 (2012), $2248-2253$.

[3] Y. Pandir, Y. Gurefe and E. Misirli, A new approach to Kudryashov's method for solving some nonlinear physical models, Int. J. Phys. Sci., 7 (2012), 2860-2866.

[4] A. Stakhov and B. Rozin, On a new class of hyperbolic functions, Chaos Soliton. Fract., 23 (2005), 379-389.

[5] E.A-B. Abdel-Salam and Z.I.A. Al-Muhiameed, Exotic localized structures based on the symmetrical Lucas function of the $(2+1)$-dimensional generalized Nizhnik-Novikov-Veselov system, Turk. J. Phys., 35 (2011), 241-256. 
[6] J. Yu, On exact solitary wave solutions to a combined KdV and $\mathrm{mKdV}$ equation, Math. Meth. Appl. Sci., 23 (2000), 1667-1670.

[7] J. H. He and X. Hong. Wu, Exp-function method for nonlinear wave equations, Chaos Soliton. Fract., 30 (2006), 700-708.

[8] A. Ebaid and E.H. Aly, Exact solutions for the transformed reduced Ostrovsky equation via the F-expansion method in terms of Weierstrass-elliptic and Jacobian-elliptic functions, Wave Motion, 49 (2012), 296-308.

[9] E. Misirli and Y. Gurefe, Exp-function method to solve the generalized Burgers-Fisher equation, Nonl. Sci. Lett. A, 1 (2010), 323-328.

[10] C.S. Liu, Exp-function method for solving nonlinear evolution equations, Math. Comput. Appl., 16 (2010), 258-266.

[11] Y. Pandir, Y. Gurefe, U. Kadak and E. Misirli, Classification of exact solutions for some nonlinear partial differential equations with generalized evolution, Abstr. Appl. Anal., 2012 (2012), 478-531.

[12] Y. Gurefe, Y. Pandir and E. Misirli, New exact solutions of stochastic KdV equation, Appl. Math. Sci., 6 (2012), 3225-3234.

[13] A.M. Wazwaz, Multiple-soliton solutions of the perturbed KdV equation, Commun. Nonl. Sci. Numer. Simulat., 15, No 11 (2010), 3270-3273.

[14] H.S. Alvaro, O.G. Hurtado and E.C. Jairo, Computing multi-soliton solutions to Caudrey-Dodd-Gibbon equation by Hirota's method, Int. J. Phys. Sci., 6 (2011), 7729-7737.

[15] Y. Zhang, S. Lai, J. Yin and Y. Wu, The application of the auxiliary equation technique to a generalized $\mathrm{mKdV}$ equation with variable coefficients, J. Comput. Appl. Math., 223 (2009), 75-85. 